\title{
Novel multihoming-based flow mobility scheme for proxy NEMO environment: A numerical approach to analyse handoff performance
}

\author{
Shayla Islam, Aisha-Hassan Abdalla, Mohammad Kamrul Hasan* \\ Department of Electrical and Computer Engineering, Faculty of Engineering, \\ International Islamic University, Kuala Lumpur, 53100, Malaysia \\ *Corresponding author, e-mail: hasankamrul@ieee.org
}

Received 22 Aug 2014

Accepted 21 May 2017

\begin{abstract}
With Network Mobility Basic Support Protocol (NEMO BSP), each communication should pass via the home agents of all mobile routers earlier reaching their destination at the time of frequent movement among the inter-technology handoff. This eventually results in performance deterioration of the real time application scenarios conducted on mobile nodes. Accordingly, applying the multihoming technique at any place, anywhere to provide uninterrupted internet connection in NEMO is becoming a significant area for current researchers. Although multiple care-of address registration between mobile routers along with its home agents can overcome some of the multihoming issues for NEMO, one still requires a dynamic flow redirection mechanism to support mobility management in NEMO. With the intention of reducing handoff delay, a novel multihoming-based flow mobility scheme on the PMIPv6 domain in NEMO (MF-PNEMO) is proposed in this paper. In addition, the performance of the MF-PNEMO scheme is evaluated through a numerical approach. The evaluation results confirms that the MF-PNEMO scheme outperforms the standard NEMO BSP as well as fast-proxy NEMO (FPNEMO) concerning handoff delay during inter-technology handoff.
\end{abstract}

KEYWORDS: NEMO BSP, MCoA, FPNEMO, inter technology

\section{INTRODUCTION}

The concept of making use of a single address introduces the hazard of network failure leading to Internet connection shut down. This occurs only if that specific single interface link fails and no options are remained to continue the connectivity. Nevertheless, users are able to switch between multiple interfaces with the introduction of multihoming technique $^{1}$. To be precise, multihoming is a networking concept which is addressed as the concurrent use of several interfaces or IP addresses on a single mobile node (MN) or mobile router (MR). It aims to improve the entire Internet connectivity as well as widens the reliability of network applications. To perform upon failure at a single attachment point, the functionality of a system component are presumed by secondary system workings if the main component is unavailable (e.g., failure). In few cases, a specific flow can be duplicated via various interfaces. Flow bindings is an addition of Multiple Care-of Address (MCoA) in Network Mobility (NEMO) that allows an MR to enclose distinct flow to a care-of address (CoA) without having an effect on other flows via the identical home address ${ }^{1,2}$. In addition, it can integrate a distinctive flow to a particular CoA exactly via correspondent node (CN) with home agent (HA) on a network-based mobility protocol (i.e., Proxy Mobile IPv6, PMIPv6) ${ }^{3,4}$. Thus it is expected to reduce handoff delay for real time application scenarios. Hence several proposals ${ }^{1-5}$ of flow mobility on PMIPv6 have already leaded to reduce registration delay during intra technology handoff. Nevertheless, adding this network-based localized protocol for inter-technology handoff in NEMO environment is still a challenging issue since both MR and its MNs should be taken into consideration $^{3}$. Additionally, a dynamic flow redirection mechanism is still required between the multiple tunnels to overcome multihoming issues in NEMO like prefix ownership and primary CoA registrations of MR with its HA which escalates the handoff delay resulting in higher packet delivery cost and extra signalling overhead ${ }^{1-3}$. To avoid this drawback, a multihoming-based flow mobility scheme on PMIPv6 in NEMO (MF-PNEMO) is proposed in this 
paper with performance estimation outcomes. It is confirmed from the outcomes that MF-PNEMO provides lower handoff delay during inter-technology handoff than that of the standard NEMO Basic Support Protocol (BSP) and Fast PMIPv6-based NEMO (FPNEMO).

The probable cases and issues for NEMO with PMIPv6 have been previously offered and studied. However, these preliminary techniques integrating PMIPv6 and NEMO suffer from extreme packet losses during the operation of handovers ${ }^{3,4}$. Accordingly, a lightweight strategy for NEMO have been proposed, which incorporates a network-based mobility protocol (i.e., PMIPv6) in NEMO (PNEMO) environment. The novelty of these paper is that MR is capable of updating the information of its existing state during handoff without creating its individual mobility signalling. The developed scheme is unaffected with link failure probability. Additionally, the lightweight mechanisms offers the improved performance by avoiding the DAD strategy for each handoff. Yet, the analytical results confirm that further investigation is required to reduce the total handoff cost in terms of location update cost as well as packet delivery cost. Furthermore, since the advanced handoff preparation strategy is applied in FPNEMO strategy, thus it decreases packet loss as well as delay during handoff within intra mobility atmosphere. Nevertheless, integrating multihoming technique with FPNEMO during inter mobility environment is yet an open research issue ${ }^{1,3,5,6}$.

Accordingly, with the intention of performing mobility as well as traffic management, a routerproxy is introduced as a central gateway inside the NEMO network in Ref. 6. In this scheme, unidirectional tunnel is used from HA to MR instead of bidirectional tunnel in order to reduce transmission delay. This scheme provides transparent and uninterrupted Internet connectivity in the perspective of handoff delay with packet loss as well as optimizes the route. However, it is still needed to focus on implementation overhead as well as security issue.

Multiple Mobile Router based scheme in NEMO (MMR-NEMO) has studied in Ref. 7, to enhance the band width, network coverage as well as reliability of a mobile network. Nevertheless, this scheme is not capable to delegate for exclusive downstream communication to MR. Hence in addition of the previous scheme, the authors has proposed a mechanism which supports upstream as well as downstream traffic ${ }^{8}$. The extended strategy permits default router assignment when the MN associates with the moving network. However, need to determine the cost of each NEMO entities for precise evaluation. Furthermore, need to focus on the signalling overhead cost due to use several MRs. Multihoming with Intelligent Make-BeforeBreak in NEMO (MIMBB-NEMO) is a new infrastructure independent handoff approach has developed in Ref. 9. MIMBB-NEMO extends MIH services to provide tunnel establishment and switching before link break. Thus the handoff is performed in background with no latency and packet loss while pingpong scenario is almost avoided. Nevertheless, needs to focus on extra signalling cost since the MIMBB-NEMO is a Global Mobility Management based scheme. Furthermore, a Multihoming-based Vertical handover scheme on PMIPv6 (MV-PMIPv6) has been proposed for node mobility in Ref. 10. According to this handover scheme, AR is replaced with a corresponding entity according to access networks. The main advantage of this scheme is that it supports 'make-before-break' handover in network layer with proxy binding update messages. However, MV-PMIPv6 mechanism should consider in NEMO environment where MR handle the mobility on behalf of all MN.

A Seamless Multi Interfaced handoff mechanism in NEMO (SMI-NEMO) network is studied in Ref. 11. To find out the best path among multiple interfaces, this paper proposed a path selection algorithm. Through analysis, it is indicated that multiple interfaced MR is capable of increasing the performance of MR movement across different network. However, it is needed to concentrate on implementation cost since to use multiple interfaces along with security issues.

In addition to that, the authors in Refs. 12-14 have proposed some proposals on PMIPv6 domain in order to support flow based routing. However, the implementation of these proposals with PMIPv6 in NEMO stills an active research area. Furthermore, analytical evaluation need to be done to determine the cost of each NEMO entities.

\section{PROPOSED MF-PNEMO SCHEME}

Different Access Technology Type (DATT) (i.e., 3G and WLAN) attached with the serving MR can attain some features, namely, increased availability, balanced traffic load with flow distribution through simultaneous connectivity during inter-technology handoff ${ }^{1,2,11}$. This is why delay during handoff are reduced $^{11}$. With the aim of taking the benefit of all multiple interfaces with better quality of service for real time applications at any time anywhere in NEMO, MF-PNEMO with predictive mode is pro- 


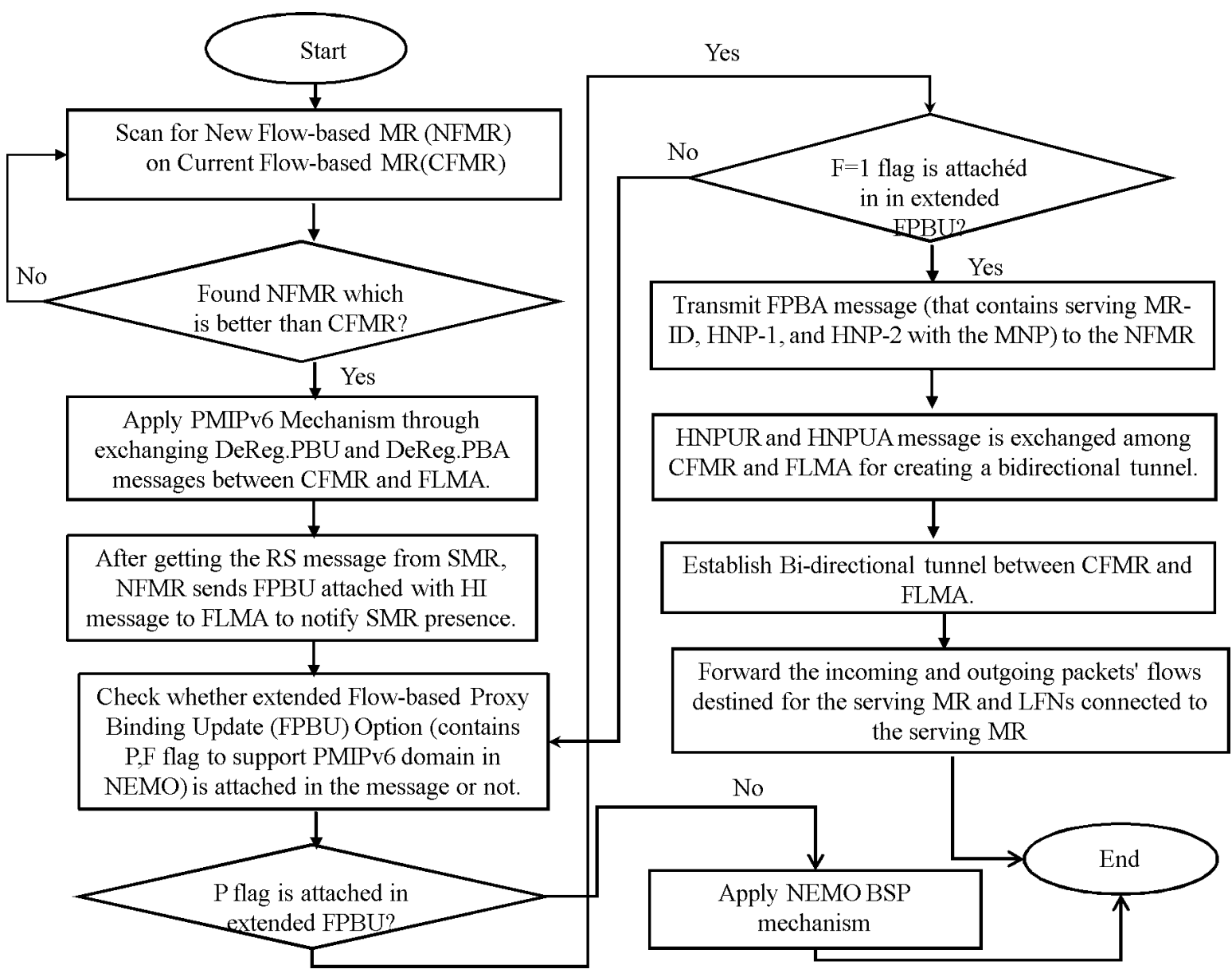

Fig. 1 Flowchart of the proposed algorithm.

posed in this paper.

\section{Serving MR, FMR and FLMA operation}

With the purpose of hiding the current multiple physical interfaces and support flow based routing, a logical interface (LI) is used by the serving $\mathrm{MR}^{2}$. LI is positioned between layer 3 and layer 2 . The $\mathrm{LI}$ can concurrently connect both Current Flow-based MR (CFMR) and New Flow-based MR (NFMR) through using multiple interfaces in the proposed scheme. Lower and upper layers send all the packets via the LI layer. The NFMR and CFMR usually run on an access router and is used as a replacement for MAG in the proposed MF-PNEMO scheme. The NFMR and CFMR are liable for identifying the serving MR's movements among DATT. According to the MF-PNEMO scheme, the Flow-based Local Mobility Anchor Point (FLMA) acts like an LMA in PMIPv6 ${ }^{1,3}$. An extension of the FLMA is made in order to permit a serving MR to register multiple proxy-CoA. Thus the FLMA is able to maintain several Binding Cache
Entries (BCE) ${ }^{4}$ for the serving MR. All entries are delegated for the serving MR's interface as well as connects with a Primary CoA (PCoA) ${ }^{3}$.

\section{Handoff Procedure of MF-PNEMO scheme}

In proposed MF-PNEMO scheme, the serving MR is capable to perform inter-technology handoff with predictive mode only. Furthermore, it is also presumed that all nodes are Local Fixed Node (LFN) beneath the serving MR and signalling message for LFN is totally controlled through the serving MR during inter mobility handoff. Otherwise, the MR performs an NEMO BSP handoff ${ }^{4}$. The flowchart of the proposed algorithm is depicted in Fig. 1. According to the MF-PNEMO scheme, the flow-based proxy binding update (FPBU) message is wrapped up with the Handover Initiation (HI) message earlier to the layer 2 (L2) handoff. Thus it is possible to reduce handoff delay for inter-technology handoff. The proposed MF-PNMEO handoff scenario with predictive mode (Fig. 1; shown in Fig. 2 accord- 


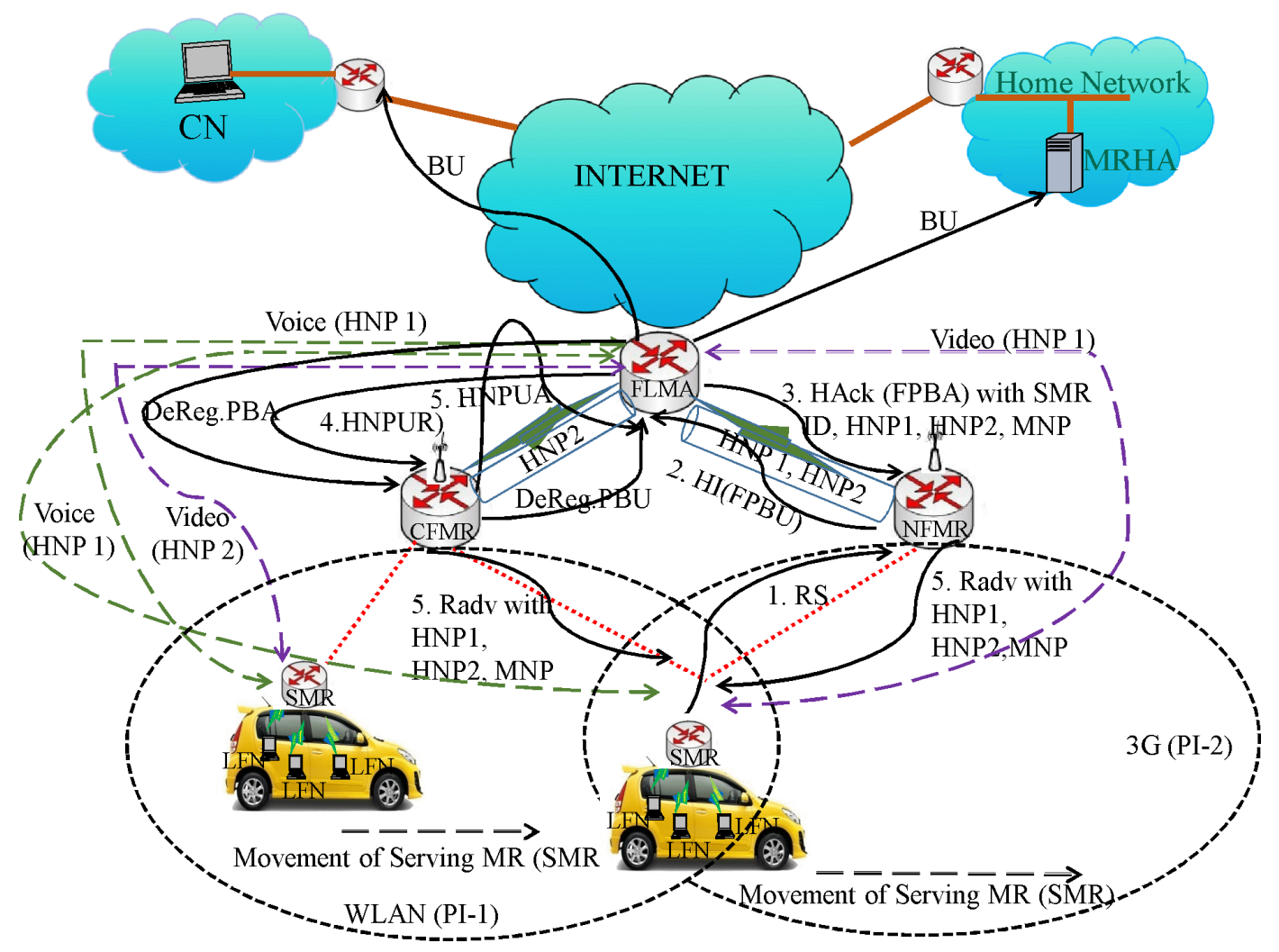

Fig. 2 Representation of proposed system Model.

ingly) is described stepwise as below:

Step 1: Initially the serving MR connects to the CFMR with the Physical Interface1 (PI 1) and assigns Home Network Prefix-1(HNP-1).

Step 2: While the serving MR comes near to the access link of NFMR, serving MR will make a decision to attach with NFMR using the Physical Interface 2 (PI 2), whereas it is still attached with CFMR by PI 1 .

Step 3: By using L2 triggering information, handover detachment of serving MR from the present access network is informed by CFMR to FLMA by exchanging de-registration proxy binding update (DeReg. PBU) message that contain the serving MR-ID and de-registration proxy binding acknowledgement (DeReg. PBA) messages.

Step 4: After sending DeReg. PBA message, FLMA needs to wait to perform handover registration for serving MR with NFMR.

Step 5: The authentication process is performed by the serving MR to access the new network after getting the L2 link up from the new access link (i.e., NFMR). NFMR will wait for router solicitation message from the newly attached serving MR on its access link, with delivering the packets to the serving MR by the NFMR.

Step 6: After getting the router solicitation message, NFMR transmits HI message with attached FPBU message (should set $F=1$ ) as shown in Fig. 3 to FLMA for notifying the presence of serving MR movement to support flow based routing on PMIPv6 domain.

Step 7: As the serving MR supports flow based routing, FLMA transmits a flow-based proxy binding acknowledgement message (contains ID of serving MR, HNP-1, and HNP-2 with the MNP) to the NFMR that supports flow bindings as well. As the bidirectional tunnel among the FLMA and NFMR is ingrained, data packets for the serving MR goes through this tunnel.

Step 8: As soon as the FLMA determines to transmit a flow via HNP, FLMA first verify whether the HNP is usable on the NFMR or not through scanning the current BCE of the serving MR. If it is usable, the FLMA should update the BCE list. If it is not usable, an HNP update request (HNPUR) as well as HNP update acknowledgement messages is exchanged among 


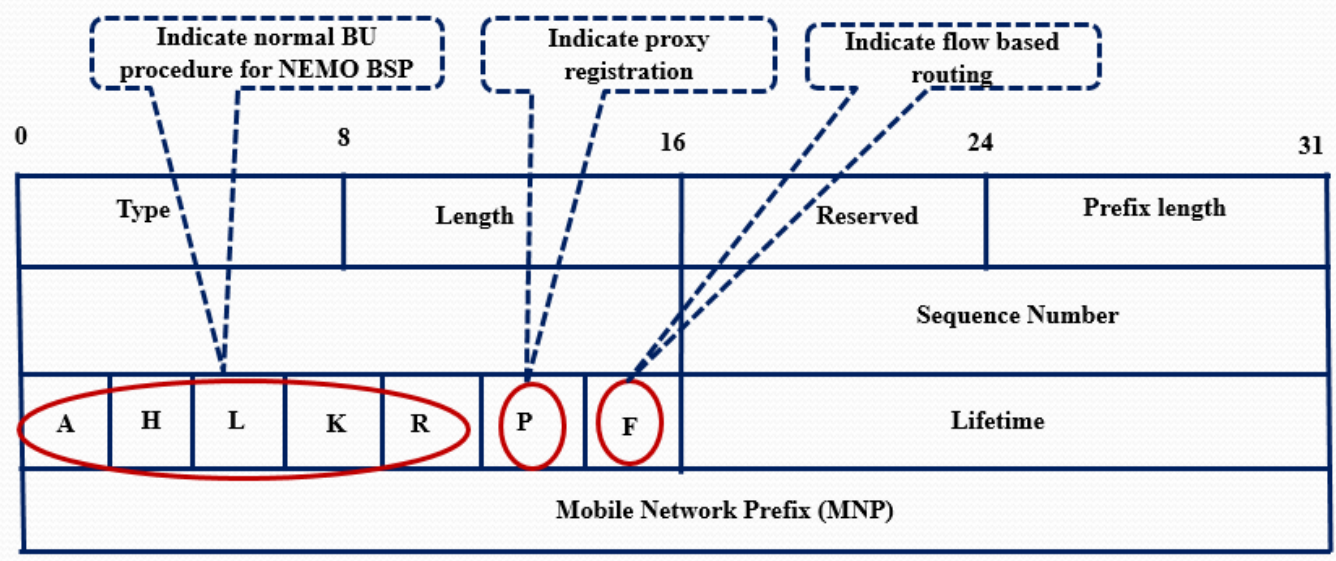

Fig. 3 Extended FPBU message.

CFMR and FLMA to inform the HNP used via that flow with creating a bidirectional tunnel. At the same time, the corresponding BCE is updated by the FLMA. As a result, the FLMA is capable of moving packet flows among multiple interfaces liberally with no additional signalling overhead. Hence, it is possible for the FLMA to pass the flow-1 which utilizes HNP1, from CFMR to NFMR directly.

\section{Extended PBU message}

Some flags should be included in FPBU message to support the proposed MF-PNEMO scheme as shown in Fig. 3. According to MF-PNEMO, $R$ bit is indicated as a flag for specifying MN mobility or an MR mobility (as shown in Fig. 3) ${ }^{3,4}$. Another flag $F$ is involved in the BU Massage to show the FLMA that the serving MR is able to maintain flow based routing. When $F$ flag is fixed to 1 , the serving MR will perform flow mobility. However, when it is set to 0 , the serving MR is not able to support flow mobility.

\section{PERFORMANCE ANALYSIS}

Due to evaluate the performance of the MF-PNEMO, numerical approaches are considered in this section. The performance evaluation of the MF-PNEMO is carried out mainly based on significant metrics like handoff delay, number of serving MRs, layer 2 handoff delay as well as cell residence time, respectively ${ }^{15,16}$. According to the proposed scheme, it is considered that all the flows are accumulated inside the equal class. Under these considerations, we evaluate total handoff delay of the MF-PNEMO scheme and then benchmark with the standard NEMO-BSP ${ }^{4,16}$ and FPNEMO ${ }^{5}$. FPNEMO ${ }^{5}$ is taken as a benchmark scheme since it is an extension of firmly-entrenched localized mobility protocol which is Proxy Mobile IPv6 ${ }^{3}$. NEMO-BSP is also an accretion of the Mobile IPv6 ${ }^{4}$. Hence FPNEMO is a suitable entrant scheme to be implemented in NEMO in order to elevate handoff performance during intertechnology handoff. The reference framework is illustrated in Fig. 4 to analyse the handoff performance of the proposed MF-PNEMO scheme.

\section{Handoff delay analysis}

An essential component of mobility management is handoff management. It aims to uphold the continuous Internet connection when the MR changes its attachment point. There are two most significant necessities for handoff management in NEMO-BSP to be fulfilled. The first requirements are seamless connectivity that must be guaranteed in terms of Mobile Router or Mobile Node Nodes location and speed. Besides, as a second requirement, it is needed to apply inter-technology handoff technique for delay sensitive applications. Time sensitive applications, like audio/video streaming is not capable of with higher handoff delays ${ }^{16,17}$. There are three types of delay during handoff in NEMO-BSP are delay for the movement detection $\Phi_{\mathrm{Q}}$, delay for the new care-of address configuration $\Phi_{\mathrm{C}}$ and delay for the registration $\Phi_{R}{ }^{16}$ expressed as

$$
\varphi_{D}=\Phi_{L}+\Phi_{\mathrm{Q}}+\Phi_{\mathrm{C}}+\Phi_{R} .
$$

In FPNEMO ${ }^{5}$ buffering mechanism is applied to receive the data packets before attaching with the new domain. The total handoff delay of the FPNEMO is expressed as $F_{D}$ :

$$
F_{D}=\Phi_{L}+2 \Phi_{\mathrm{T}}+\left(H_{\mathrm{M}}+H_{L}\right) \Phi_{\mathrm{W}} \text {, }
$$




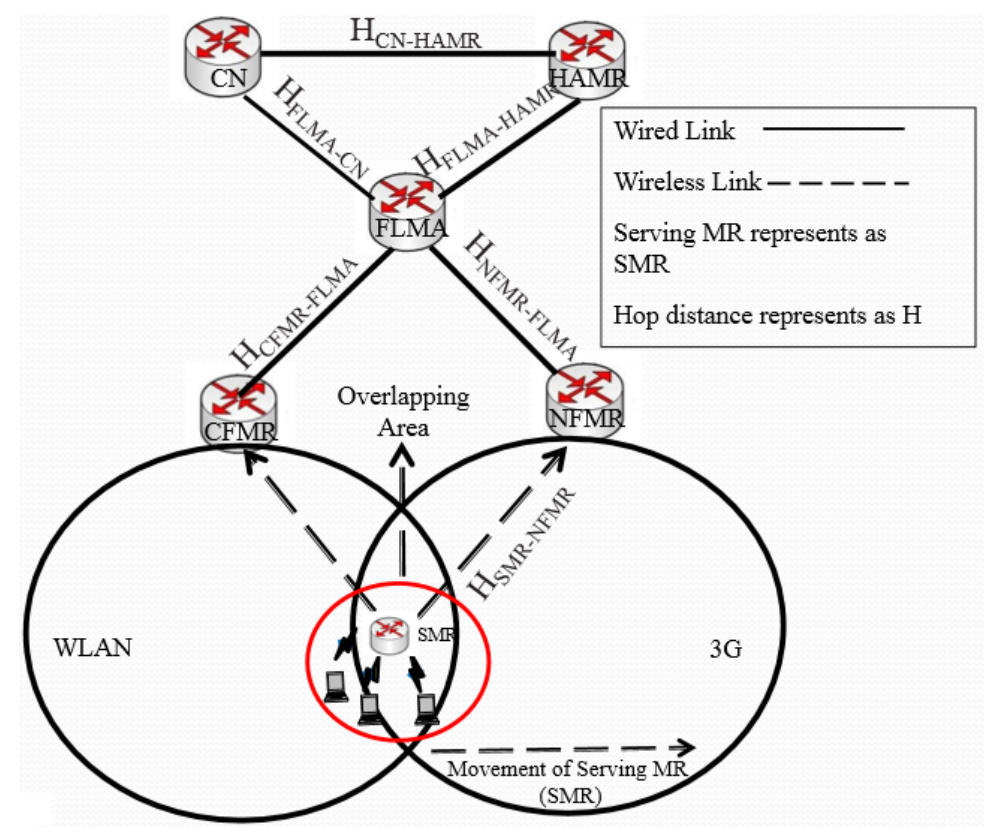

Fig. 4 Numerical framework of the MF-PNEMO scheme.

where $F_{D}$ represents the delays in the FPNEMO. Wired link delay and wireless link delay are denoted by $\Phi_{\mathrm{T}}$ and $\Phi_{\mathrm{W}}$, respectively. The hop distances in between the MAGs and the LMA as well as MAG are represented as $H_{\mathrm{M}}$ and $H_{L}$, respectively. According to the proposed MF-PNEMO scheme, the time discrepancy between the very last data packet received at the previous link and the first data packet received on the new link can be denoted by total handoff delay. In case of real time application, this delay is a crucial performance metrics. It is assumed that the time for new care-of address configuration as well as layer 2 handoff are steady due to analyse the influence of signalling techniques to the handoff delay. According to the proposed MF-PNEMO scheme, it is not required to send the $\mathrm{BU}$ message via the wireless link. Hence handoff delay is calculated as $\Phi_{D}$ :

$$
\begin{gathered}
\Phi_{D}=D_{L} \frac{L_{\mathrm{M}}}{B_{\mathrm{W}}}+\Phi_{\mathrm{W}}+D_{H} \frac{L_{H}+L_{\mathrm{A}}}{B_{\mathrm{W}}}+\left(\Phi_{\mathrm{W}}\right) \\
\varphi_{\mathrm{DP}}=\Phi_{L}+\max \left(\Phi_{\mathrm{W}}, \Phi_{D}\right) .
\end{gathered}
$$

Here, from (3) and (4), $\varphi_{D P}$ represents the delays in the MF-PNEMO. The hop distances in between the FMRs and the FLMA as well as FMR are identified as $D_{L}$ and $D_{H}$, respectively. Hence the relative Handoff Delay Gain of MF-PNEMO and FPNEMO can be defined as $\kappa_{D}$ :

$$
\kappa_{D}=\frac{F_{D}}{\varphi_{\mathrm{DP}}}
$$

Table 1 Parameters for handoff delay analysis ${ }^{5,15,16}$.

\begin{tabular}{ll}
\hline Parameter & Value \\
\hline Hope distance among FMRs & 1 \\
Hope distance among FMR and FLMA & 5 \\
Layer 2 handoff time & $100 \mathrm{~ms}$ \\
Length of HI message & 52 bytes \\
Length of FPBU message & 76 bytes \\
Length of HNPUR message & 112 bytes \\
\hline
\end{tabular}

\section{Result analysis}

The values used as system parameters are listed in Table $1^{5,15,16}$.

\section{Total handoff delay}

The variation of total handoff delay for MF-PNEMO, FPNEMO, and NEMO-BSP are illustrated in Fig. 5 as a function of the different number of serving MR along with average cell residence time. It is indicated from Fig. 5 that, handoff delays of all schemes escalates linearly with raising the number of serving MR due to send greater number of location updates along with much more processing cost at HA for NEMO BSP, at LMA for FPNEMO and at FLMA for MF-PNEMO scheme. As depicted in Fig. 5, handoff delay is much higher in the traditional scheme (i.e., NEMO-BSP and FPNEMO) since to swap a number of signalling message via the wireless link as well as to establish multiple tunnels. Furthermore, cell res- 


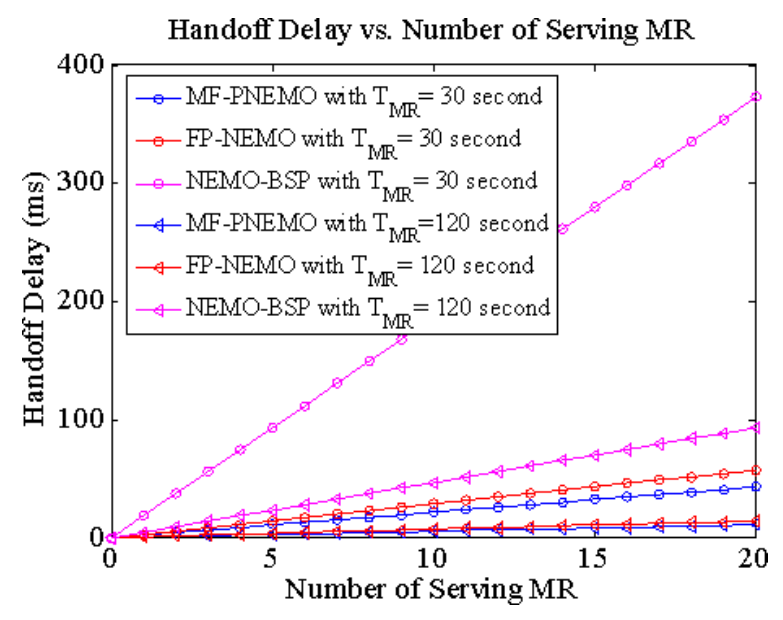

Fig. 5 Handoff delay of MF-PNEMO, FPNEMO, and NEMO-BSP scheme.

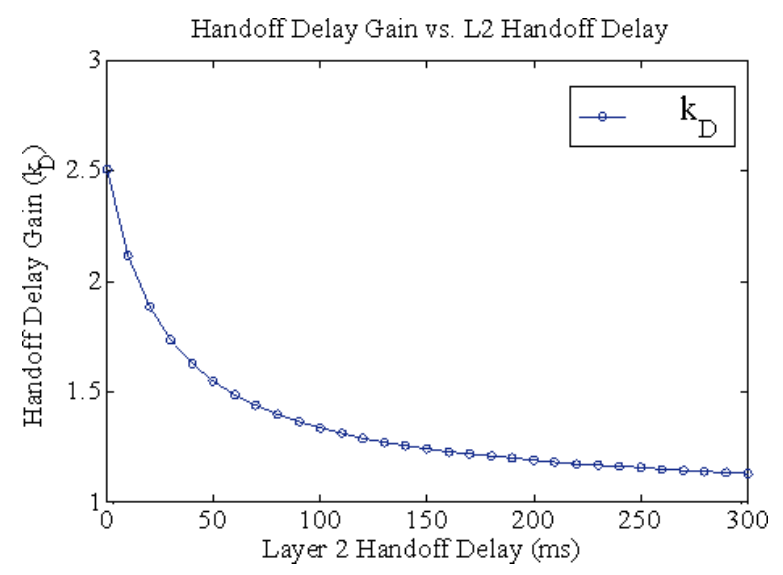

Fig. 6 Gain of MF-PNEMO scheme against FPNEMO.

idence time decrement has led the handoff delay to rise abruptly for NEMO BSP which in turn increases the total handoff delay significantly as shown in Fig. 5. As there is a relation between $\mathrm{L} 2$ delay and its effect on wireless link, Fig. 6 shows the total handoff gain of the MF-PNEMO scheme as well as FPNEMO. The gain of handoff delay rises when L2 handoff delay declines as it is noticed from Fig. 6 .

As multiple interfaces is equipped in the proposed MF-PNEMO scheme. Hence the L2 delay is become smaller whereas the L2 delay for NEMO BSP as well as FPNEMO is not shorter due to use single interface. Hence it is summed up from Figs. 5 and 6 that the proposed MF-PNEMO scheme has lower handoff delay than NEMO-BSP and FPNEMO regardless of increasing number of serving $M R$ and L2 delay time.

\section{CONCLUSIONS}

This paper proposes a novel Multihoming-based Flow mobility scheme on PMIPv6 domain in NEMO (MF-PNEMO) to support mobility management. The major contribution of this paper is to reduce handoff delay by including PMIPv6 in NEMO. Thus MFPNEMO scheme eradicates the necessity to apprise the current position of the serving MR to its HA before travelling to multiple access routers. In addition to that, through applying a dynamic flow redirection mechanism, it is feasible to reduce handoff delay which leads to provide a better quality of service. The performance of the MF-PNEMO scheme is evaluated via numerical analysis. Hence it can be summed up from analytical analysis that the handoff delay of NEMO-BSP and FPNEMO shows superior than MF-PNEMO scheme due to multiple tunnelled packets need to be routed from $\mathrm{CN}$ to the MR via the HA of the MR in NEMO BSP as well as lack of multihoming technique. However, experimental test bed is needed to include for more precise evaluation of the proposed MF-PNEMO scheme as future recommendation.

Acknowledgements: A special thanks to the Government of Malaysia, through Ministry of education for the education sponsorship. The authors would also like to thank the Research Management Centre at the International Islamic University Malaysia for the grant sponsorship in part.

\section{REFERENCES}

1. Sarikaya B (2012) PMIPv6 multihoming support extensions for flow mobility. IETF Internet-Draft draftsarikaya-netext-flowmob-ext-00, Internet Engineering Task Force, [work in progress].

2. Tsirtsis G, Soliman H, Montavont N, Giaretta G, Kuladinithi K (2011) Flow bindings in Mobile IPv6 and Network Mobility (NEMO) basic support. IETF Request for Comments RFC 6089, Internet Engineering Task Force.

3. Bernardos CJ, Calderon M, Soto I (2012) PMIPv6 and network mobility problem statement. IETF InternetDraft draft-bernardos-netext-pmipv6-nemo-ps-02, Internet Engineering Task Force, [work in progress].

4. Devarapalli V, Wakikawa R, Petrescu A, Thubert P (2005) Network Mobility (NEMO) basic support protocol. IETF Request for Comments RFC 3963, Internet Engineering Task Force.

5. Lee JH, Ernst T, Chilamkurti N (2012) Performance analysis of PMIPv6-based NEtwork MObility for Intelligent Transportation Systems. IEEE Trans Veh Tech 61, 74-85. 
6. Slimane Z, Feham M, Abdelmalek A (2010) A seamless and transparent MN-proxy based mobility support for $(n, n, 1)$ multihomed NEMO model. Int $J$ Comput Sci Netw Secur 10, 306-13.

7. Kuntz R, Montavont J, Noel T (2008) Multiple mobile routers in NEMO: How Neighbor Discovery can assist default router selection. In: IEEE 19th International Symposium on Personal, Indoor and Mobile Radio Communications, pp 1-6.

8. Kuntz R, Montavont J, Noel T (2013) Multihoming in ipv6 mobile networks: Progress, challenges, and solutions. IEEE Comm Mag 51, 128-35.

9. Slimane Z, Feham M, Abdelmalek A (2012) Seamless infrastructure independent multi homed NEMO handoff using effective and timely ieee 802.21 $\mathrm{MIH}$ triggers. Int J Wireless Mobile Network 4, 119-39.

10. Park HD, Park KN (2013) A multihoming-based vertical handover scheme. In: Kim KJ, Chung KY (eds) IT Convergence and Security 2012, Lecture Notes in Electrical Engineering vol 215, Springer, Dordrecht, pp 749-54.

11. Chen X, Zhang H, Chang YC, Chao HC (2010) Experimentation and performance analysis of multiinterfaced mobile router scheme. Simulat Model Pract Theor 18, 407-15.

12. Trung TM, Han YH, Choi HY, Geun HY (2011) A design of network-based flow mobility based on proxy mobile IPv6. In: 2011 IEEE Conference on Computer Communications Workshops (INFOCOM WKSHPS), pp 373-8.

13. Melia T, Bernardos CJ, De la Oliva A, Giust F, Calderon M (2011) IP flow mobility in PMIPv6 based networks: Solution design and experimental evaluation. Wireless Pers Comm 61, 603-27.

14. Choi HY, Min SG, Han YH, Koodli R (2012) Design and simulation of a flow mobility scheme based on Proxy Mobile IPv6. J Inform Process Syst 8, 603-20.

15. Hossain MS, Atiquzzaman M, Ivancic W (2011) Cost analysis of mobility management entities of SINEMO. In: 2011 IEEE International Conference on Communications (ICC), pp 1-5.

16. Huang Z, Yang Y, Hu H, Lin KC (2010) A fast handover scheme based on multiple mobile router cooperation for a train-based mobile network. Int $J$ Model Ident Contr 10, 202-12.

17. Chen Y, Farley T, Ye N (2004) QoS requirements of network applications on the Internet. Inform Knowl Syst Manag 4, 55-76. 